\title{
Global Existence and Uniqueness of Strong Solutions for the Magnetohydrodynamic Equations
}

\author{
Jianwen Zhang \\ School of Mathematical Sciences, Xiamen University, Xiamen 361005, China \\ Correspondence should be addressed to Jianwen Zhang, jwzhang@xmu.edu.cn
}

Received 21 June 2007; Accepted 5 October 2007

Recommended by Colin Rogers

This paper is concerned with an initial boundary value problem in one-dimensional magnetohydrodynamics. We prove the global existence, uniqueness, and stability of strong solutions for the planar magnetohydrodynamic equations for isentropic compressible fluids in the case that vacuum can be allowed initially.

Copyright (c) 2008 Jianwen Zhang. This is an open access article distributed under the Creative Commons Attribution License, which permits unrestricted use, distribution, and reproduction in any medium, provided the original work is properly cited.

\section{Introduction}

Magnetohydrodynamics (MHD) concerns the motion of a conducting fluid in an electromagnetic field with a very wide range of applications. The dynamic motion of the fluids and the magnetic field strongly interact each other, and thus, both the hydrodynamic and electrodynamic effects have to be considered. The governing equations of the plane magnetohydrodynamic compressible flows have the following form (see, e.g., [1-5]):

$$
\begin{gathered}
\rho_{t}+(\rho u)_{x}=0 \\
(\rho u)_{t}+\left(\rho u^{2}+p+\frac{1}{2}|\mathbf{b}|^{2}\right)_{x}=\left(\lambda u_{x}\right)_{x^{\prime}} \\
(\rho \mathbf{w})_{t}+(\rho u \mathbf{w}-\mathbf{b})_{x}=\left(\mu \mathbf{w}_{x}\right)_{x^{\prime}} \\
\mathbf{b}_{t}+(u \mathbf{b}-\mathbf{w})_{x}=\left(v \mathbf{b}_{x}\right)_{x^{\prime}} \\
(\rho e)_{t}+(\rho e u)_{x}-\left(\kappa \theta_{x}\right)_{x}=\lambda u_{x}^{2}+\mu\left|\mathbf{w}_{x}\right|^{2}+v\left|\mathbf{b}_{x}\right|^{2}-p u_{x}
\end{gathered}
$$

where $\rho$ denotes the density of the fluid, $u \in \mathbb{R}$ the longitudinal velocity, $\mathbf{w}=\left(w_{1}, w_{2}\right) \in \mathbb{R}^{2}$ the transverse velocity, $\mathbf{b}=\left(b_{1}, b_{2}\right) \in \mathbb{R}^{2}$ the transverse magnetic field, $\theta$ the temperature, 
$p=p(\rho, \theta)$ the pressure, and $e=e(\rho, \theta)$ the internal energy; $\lambda$ and $\mu$ are the bulk and shear viscosity coefficients, $v$ is the magnetic viscosity, $\kappa$ is the heat conductivity. Notice that the longitudinal magnetic field is a constant which is taken to be identically one in (1.1).

The equations in (1.1) describe the macroscopic behavior of the magnetohydrodynamic flow. This is a three-dimensional magnetohydrodynamic flow which is uniform in the transverse directions. There is a lot of literature on the studies of MHD by many physicists and mathematicians because of its physical importance, complexity, rich phenomena, and mathematical challenges, see [1-14] and the references cited therein. We mention that, when $\mathbf{b}=0$, the system (1.1) reduces to the one-dimensional compressible Navier-Stokes equations for the flows between two parallel horizontal plates (see, e.g., [15]).

In this paper, we focus on a simpler case of (1.1), namely, we consider the magnetohydrodynamic equations for isentropic compressible fluids. Thus, instead of the equations in (1.1), we will study the following equations:

$$
\begin{gathered}
\rho_{t}+(\rho u)_{x}=0 \\
(\rho u)_{t}+\left(\rho u^{2}+p+\frac{1}{2}|\mathbf{b}|^{2}\right)_{x}=\left(\lambda u_{x}\right) x \\
(\rho \mathbf{w})_{t}+(\rho u \mathbf{w}-\mathbf{b})_{x}=\left(\mu \mathbf{w}_{x}\right)_{x^{\prime}} \\
\mathbf{b}_{t}+(u \mathbf{b}-\mathbf{w})_{x}=\left(v \mathbf{b}_{x}\right)_{x^{\prime}}
\end{gathered}
$$

where $p=R \rho^{\gamma}$ with $\gamma \geq 1$ being the adiabatic exponent and $R>0$ being the gas constant. We will study the initial boundary value problem of (1.2)-(1.5) in a bounded spatial domain $\Omega=(0,1)$ (without loss of generality) with the initial-boundary data:

$$
\begin{gathered}
(\rho, \rho u, \rho \mathbf{w}, \mathbf{b})(x, 0)=\left(\rho_{0}, m_{0}, \mathbf{n}_{0}, \mathbf{b}_{0}\right)(x), \quad x \in \Omega, \\
\left.(u, \mathbf{w}, \mathbf{b})\right|_{x=0,1}=0
\end{gathered}
$$

where the initial data $\rho_{0} \geq 0, m_{0}, \mathbf{n}_{0}, \mathbf{b}_{0}$ satisfy certain compatibility conditions as usual and some additional assumptions below, and $m_{0}=\mathbf{n}_{0}=0$ whenever $\rho_{0}=0$. Here the boundary conditions in (1.7) mean that the boundary is nonslip and impermeable.

The purpose of the present paper is to study the global existence and uniqueness of strong solutions of problem (1.2)-(1.7). The important point here is that initial vacuum is allowed; that is, the initial density $\rho_{0}$ may vanish in an open subset of the space-domain $\Omega=(0,1)$, which evidently makes the existence and regularity questions more difficult than the usual case that the initial density $\rho_{0}$ has a positive lower bound. For the latter case, one can show the global existence of unique strong solution of this initial boundary value problem in a similar way as that in $[3,9,14]$. The strong solutions of the Navier-Stokes equations for isentropic compressible fluids in the case that initial vacuum is allowed have been studied in $[16,17]$. In this paper, we will use some ideas developed in $[16,17]$ and extend their results to the problems (1.2)-(1.7). However, because of the additional nonlinear equations and the nonlinear terms induced by the magnetic field $\mathbf{b}$, our problem becomes a bit more complicated.

Our main result in this paper is given by the following theorem (the notations will be defined at the end of this section). 
Theorem 1.1. Assume that $\rho_{0}, m_{0}=\rho_{0} u_{0}, \mathbf{n}_{0}=\rho_{0} \mathbf{w}_{0}$, and $\mathbf{b}_{0}$ satisfy the regularity conditions:

$$
\rho_{0} \in \mathscr{H}^{1}, \quad \rho_{0} \geq 0, \quad\left(u_{0}, \mathbf{w}_{0}\right) \in \mathscr{H}_{0}^{1} \cap \mathscr{\ell}^{2}, \quad \mathbf{b}_{0} \in \mathscr{H}_{0}^{1}
$$

Assume also that the following compatibility conditions hold for the initial data:

$$
\begin{gathered}
\lambda u_{0 x x}-\left(R \rho_{0}^{\gamma}+\frac{1}{2}\left|\mathbf{b}_{0}\right|^{2}\right)_{x}=\rho_{0}^{1 / 2} f \quad \text { for some } f \in \mathcal{L}^{2}(\Omega) \\
\mu \mathbf{w}_{0 x x}+\mathbf{b}_{0 x}=\rho_{0}^{1 / 2} \mathbf{g} \quad \text { for some } \mathbf{g} \in \mathcal{L}^{2}(\Omega)
\end{gathered}
$$

Then there exists a unique global strong solution $(\rho, u, \mathbf{w}, \mathbf{b})$ to the initial boundary value problem (1.2)-(1.7) such that for all $T \in(0, \infty)$,

$$
\begin{gathered}
\rho \in \mathcal{L}^{\infty}\left(0, T ; \mathscr{L}^{1}\right), \quad(u, \mathbf{w}) \in \mathcal{L}^{\infty}\left(0, T ; \mathscr{L}_{0}^{1} \cap \mathscr{L}^{2}\right), \quad \mathbf{b} \in \mathcal{L}^{\infty}\left(0, T ; \mathscr{L}_{0}^{1}\right), \\
\left(\rho_{t^{\prime}} \sqrt{\rho} u_{t}, \sqrt{\rho} \mathbf{w}_{t}\right) \in \mathcal{L}^{\infty}\left(0, T ; \mathcal{L}^{2}\right), \quad\left(u_{t}, \mathbf{w}_{t}\right) \in \mathcal{L}^{2}\left(0, T ; \mathscr{L}^{1}\right), \quad\left(\mathbf{b}_{t}, \mathbf{b}_{x x}\right) \in \mathcal{L}^{2}\left(0, T ; \mathcal{L}^{2}\right) .
\end{gathered}
$$

Remark 1.2. The compatibility conditions given by (1.9), (1.10) play an important role in the proof of uniqueness of strong solutions. Similar conditions were proposed in [16-18] when the authors studied the global existence and uniqueness of solutions of the Navier-Stokes equations for isentropic compressible fluids. In fact, one also can show the global existence of weak solutions without uniqueness if the compatibility conditions (1.9), (1.10) are not valid.

We will prove the global existence and uniqueness of strong solutions in Sections 3 and 4 , respectively, while Section 2 is devoted to the derivation of some a priori estimates.

We end this section by introducing some notations which will be used throughout the paper. Let $\mathcal{W}^{m, p}(\Omega)$ denote the usual Sobolev space, and $\mathcal{W}^{m, 2}(\Omega)=\mathscr{L}^{m}(\Omega), \mathcal{W}^{0, p}(\Omega)=\mathcal{L}^{p}(\Omega)$. For simplicity, we denote by $C$ the various generic positive constants depending only on the data and $T$, and use the following abbreviation:

$$
\mathcal{L}^{q}\left(0, T ; \mathcal{W}^{m, p}\right) \equiv \mathcal{L}^{q}\left(0, T ; \mathcal{W}^{m, p}(\Omega)\right), \quad \mathcal{L}^{p} \equiv \mathcal{L}^{p}(\Omega), \quad\|\cdot\|_{p} \equiv\|\cdot\|_{\mathcal{L}^{p}(\Omega)} .
$$

\section{A priori estimates}

This section is devoted to the derivation of a priori estimates of $(\rho, u, \mathbf{w}, \mathbf{b})$. We begin with the observation that the total mass is conserved. Moreover, if we multiply (1.3), (1.4), and (1.5) by $u, \mathbf{w}$, and $\mathbf{b}$, respectively, and sum up the resulting equations, we have by using (1.2) that

$$
\begin{gathered}
\left(\frac{1}{2} \rho\left(u^{2}+|\mathbf{w}|^{2}\right)+\frac{1}{2}|\mathbf{b}|^{2}\right)_{t}+\left(\frac{1}{2} \rho u\left(u^{2}+|\mathbf{w}|^{2}\right)+u|\mathbf{b}|^{2}-\mathbf{w} \cdot \mathbf{b}\right)_{x}+u p_{x} \\
=\left(\lambda u u_{x}+\mu \mathbf{w} \cdot \mathbf{w}_{x}+v \mathbf{b} \cdot \mathbf{b}_{x}\right)_{x}-\left(\lambda u_{x}^{2}+\mu\left|\mathbf{w}_{x}\right|^{2}+v\left|\mathbf{b}_{x}\right|^{2}\right)
\end{gathered}
$$

Integrating (1.2) and (2.1) over $(0, t) \times \Omega$, we arrive at our first lemma. 
Lemma 2.1. For any $t \in(0, T)$, one has

$$
\begin{aligned}
& \int_{\Omega} \rho(x, t) d x=\int_{\Omega} \rho_{0}(x) d x \leq C, \\
& \int_{\Omega}\left(G(\rho)+\frac{1}{2} \rho\left(u^{2}+|\mathbf{w}|^{2}\right)+\frac{1}{2}|\mathbf{b}|^{2}\right)(x, t) d x+\int_{0}^{t} \int_{\Omega}\left(\lambda u_{x}^{2}+\mu\left|\mathbf{w}_{x}\right|^{2}+v\left|\mathbf{b}_{x}\right|^{2}\right)(x, s) d x d s \leq C,
\end{aligned}
$$

where $G(\rho)$ is the nonnegative function defined by

$$
G(\rho)= \begin{cases}\frac{R \rho^{\gamma}}{\gamma-1} & \text { if } \gamma>1 \\ R(\rho \ln \rho-\rho+1) & \text { if } \gamma=1\end{cases}
$$

The next lemma gives us an upper bound of the density $\rho(x, t)$, which is crucial for the proof of Theorem 1.1 .

Lemma 2.2. For any $(x, t) \in Q_{T}:=\Omega \times(0, T), \rho(x, t) \leq C$ holds.

Proof. Notice that (1.3) can be rewritten as

$$
(\rho u)_{t}=\left(\lambda u_{x}-\rho u^{2}-p-\frac{|\mathbf{b}|^{2}}{2}\right)_{x}
$$

Set

$$
\psi(x, t):=\int_{0}^{t}\left(\lambda u_{x}-\rho u^{2}-p-\frac{1}{2}|\mathbf{b}|^{2}\right)(x, s) d s+\int_{0}^{x} m_{0}(\zeta) d \zeta,
$$

from which and (2.4), we find that $\psi$ satisfies

$$
\psi_{x}=\rho u, \quad \psi_{t}=\lambda u_{x}-\rho u^{2}-p-\frac{1}{2}|\mathbf{b}|^{2},\left.\quad \psi\right|_{t=0}=\int_{0}^{x} m_{0}(\zeta) d \zeta .
$$

In view of Lemma 2.1 and (2.6), we have by using Cauchy-Schwarz's inequality that

$$
\left\|\psi_{x}\right\|_{\mathcal{L}^{\infty}\left(0, T ; \mathcal{L}^{1}\right)} \leq C, \quad\left|\int_{\Omega} \psi(x, t) d x\right| \leq C,
$$

which imply

$$
\|\psi\|_{\mathcal{L}^{\infty}((0, T) \times \Omega)} \leq\left\|\psi_{x}\right\|_{\mathcal{L}^{\infty}\left(0, T ; \mathcal{L}^{1}\right)}+\left|\int_{\Omega} \psi(x, t) d x\right| \leq C .
$$

Letting $D_{t}:=\partial_{t}+u \partial_{x}$ denote the material derivative and choosing $F=\exp (\psi / \lambda)$, we obtain after a straightforward calculation that

$$
D_{t}(\rho F):=\partial_{t}(\rho F)+u \partial_{x}(\rho F)=-\frac{1}{\lambda}\left(p+\frac{|\mathbf{b}|^{2}}{2}\right) \rho F \leq 0,
$$

which, together with (2.8), yields Lemma 2.2 immediately. 
Jianwen Zhang

To be continued, we need the following lemma because of the effect of magnetic field $\mathbf{b}$.

Lemma 2.3. The magnetic field $\mathbf{b}$ satisfies the following estimates:

$$
\sup _{0 \leq t \leq T}\left(\|\mathbf{b}(t)\|_{\mathcal{L}^{\infty}}+\left\|\mathbf{b}_{x}(t)\right\|_{\mathcal{L}^{2}}\right)+\left\|\mathbf{b}_{t}\right\|_{\mathcal{L}^{2}\left(0, T ; \mathcal{L}^{2}\right)} \leq C, \quad\left\|\mathbf{b}_{x x}\right\|_{\mathcal{L}^{2}\left(0, T ; \mathcal{L}^{2}\right)} \leq C .
$$

Proof. Multiplying (1.5) by $\mathbf{b}_{t}$ and integrating over $(0, t) \times \Omega$, we have

$$
\begin{aligned}
& \frac{1}{4} \int_{0}^{t} \int_{\Omega}\left|\mathbf{b}_{t}\right|^{2}(x, s) d x d s+\frac{v}{2} \int_{\Omega}\left|\mathbf{b}_{x}\right|^{2}(x, t) d x \\
& \quad \leq \frac{v}{2} \int_{\Omega}\left|\mathbf{b}_{0 x}\right|^{2}(x) d x+\int_{0}^{t} \int_{\Omega}\left(u^{2}\left|\mathbf{b}_{x}\right|^{2}+u_{x}^{2}|\mathbf{b}|^{2}+\left|\mathbf{w}_{x}\right|^{2}\right)(x, s) d x d s \\
& \quad \leq C+2 \int_{0}^{t}\left(\int_{\Omega} u_{x}^{2}(x, s) d x\right)\left(\int_{\Omega}\left|\mathbf{b}_{x}\right|^{2}(x, s) d x\right) d s
\end{aligned}
$$

where we have used Cauchy-Schwarz's inequality, Lemma 2.1, and the following inequalities:

$$
\max _{x \in \Omega} u^{2}(\cdot, s) \leq\left\|u_{x}(s)\right\|_{\mathcal{L}^{2},}^{2}, \quad \max _{x \in \Omega}|\mathbf{b}(\cdot, s)|^{2} \leq\left\|\mathbf{b}_{x}(s)\right\|_{\mathcal{L}^{2}}^{2} \text {. }
$$

Since $\left\|u_{x}\right\|_{\mathcal{L}^{2}\left(0, T ; \mathfrak{L}^{2}\right)} \leq C$ because of Lemma 2.1, we thus obtain the first inequality indicated in this lemma from (2.11) by applying Gronwall's lemma and then Sobolev's inequality.

To prove the second part, we multiply (1.5) by $\mathbf{b}_{x x}$ and integrate the resulting equation over $(0, T) \times \Omega$ to deduce that

$$
\begin{aligned}
& \int_{0}^{T} \int_{\Omega}\left|\mathbf{b}_{x x}\right|^{2}(x, t) d x d t \\
& \quad \leq C \int_{0}^{T} \int_{\Omega}\left(\left|\mathbf{b}_{t}\right|^{2}+\left|\mathbf{w}_{x}\right|^{2}+u_{x}^{2}|\mathbf{b}|^{2}+u^{2}\left|\mathbf{b}_{x}\right|^{2}\right)(x, t) d x d t \\
& \quad \leq C+C \sup _{t \in(0, T)}\|\mathbf{b}(t)\|_{\mathcal{L}^{\infty}}^{2} \int_{0}^{T}\left\|u_{x}(t)\right\|_{\mathcal{L}^{2}}^{2} d t+C \int_{0}^{T}\left\|u_{x}(t)\right\|_{\mathcal{L}^{2}}^{2}\left\|\mathbf{b}_{x}(t)\right\|_{\mathcal{L}^{2}}^{2} d t \\
& \quad \leq C+C \sup _{t \in(0, T)}\left(\|\mathbf{b}(t)\|_{\mathcal{L}^{\infty}}^{2}+\left\|\mathbf{b}_{x}(t)\right\|_{\mathcal{L}^{2}}^{2}\right) \int_{0}^{T}\left\|u_{x}(t)\right\|_{\mathcal{L}^{2}}^{2} d t \leq C,
\end{aligned}
$$

where we have used Cauchy-Schwarz's inequality, Sobolev's inequality (2.12), Lemma 2.1, and the first part of the lemma. This completes the proof of Lemma 2.3.

Lemma 2.4. The following estimates hold for the velocity $(u, \mathbf{w})$ :

$$
\begin{aligned}
& \sup _{0 \leq t \leq T}\left(\|u(t)\|_{\mathcal{L}^{\infty}}+\left\|u_{x}(t)\right\|_{\mathcal{L}^{2}}\right)+\left\|\sqrt{\rho} u_{t}\right\|_{\mathcal{L}^{2}\left(0, T ; \mathcal{L}^{2}\right)} \leq C, \\
& \sup _{0 \leq t \leq T}\left(\|\mathbf{w}(t)\|_{\mathcal{L}^{\infty}}+\left\|\mathbf{w}_{x}(t)\right\|_{\mathcal{L}^{2}}\right)+\left\|\sqrt{\rho} \mathbf{w}_{t}\right\|_{\mathcal{L}^{2}\left(0, T ; \mathcal{L}^{2}\right)} \leq C .
\end{aligned}
$$


Proof. Multiplying (1.3) by $\boldsymbol{u}_{t}$ and then integrating over $\Omega$, by Young's inequality we obtain

$$
\frac{\lambda}{2} \frac{d}{d t} \int_{\Omega} u_{x}^{2} d x+\frac{1}{2} \int_{\Omega} \rho u_{t}^{2} d x \leq \frac{1}{2} \int_{\Omega} \rho u^{2} u_{x}^{2} d x+\int_{\Omega} p u_{t x} d x d t+\frac{1}{2} \int_{\Omega}|\mathbf{b}|^{2} u_{x t} d x .
$$

It follows from (1.2) and (1.3) that

$$
\begin{aligned}
\int_{\Omega} p u_{t x} d x= & \frac{d}{d t} \int_{\Omega} p u_{x} d x-\frac{R}{2 \lambda} \frac{d}{d t} \int_{\Omega} \rho^{\gamma+1} u^{2} d x-\frac{R(\gamma-2)}{2 \lambda} \int_{\Omega} \rho^{\gamma+1} u^{2} u_{x} d x \\
& +\frac{1}{2 \lambda} \int_{\Omega} p^{2} u_{x} d x-\frac{1}{\lambda} \int_{\Omega} p u\left(\rho u u_{x}+\mathbf{b} \cdot \mathbf{b}_{x}\right) d x+(\gamma-1) \int_{\Omega} p u_{x}^{2} d x \\
\frac{1}{2} \int_{\Omega}|\mathbf{b}|^{2} u_{x t} d x= & \frac{1}{2} \frac{d}{d t} \int_{\Omega}|\mathbf{b}|^{2} u_{x} d x-\int_{\Omega} \mathbf{b} \cdot \mathbf{b}_{t} u_{x} d x .
\end{aligned}
$$

Thus, inserting (2.16) into (2.15), and integrating over $(0, t)$, we see that

$$
\begin{aligned}
& \int_{\Omega} u_{x}^{2}(x, t) d x+\int_{0}^{t} \int_{\Omega} \rho u_{t}^{2} d x d s \\
& \leq C+C \int_{\Omega}\left(p\left|u_{x}\right|+\rho^{\gamma+1} u^{2}+|\mathbf{b}|^{2}\left|u_{x}\right|\right)(x, t) d x \\
& \quad+C \int_{0}^{t} \int_{\Omega}\left(\rho u^{2} u_{x}^{2}+\rho^{\gamma+1} u^{2}\left|u_{x}\right|+p^{2}\left|u_{x}\right|+\left|p u \mathbf{b} \cdot \mathbf{b}_{x}\right|+p u_{x}^{2}+\left|\mathbf{b} \cdot \mathbf{b}_{t} u_{x}\right|\right) d x d s
\end{aligned}
$$

where the terms on the right-hand side can be bounded by using Lemmas 2.1-2.3 as follows:

$$
\begin{gathered}
\int_{\Omega}\left(p\left|u_{x}\right|+\rho^{\gamma+1} u^{2}+|\mathbf{b}|^{2}\left|u_{x}\right|\right)(t) d x \leq C(\delta)+\delta \int_{\Omega} u_{x}^{2}(t) d x, \quad \delta>0, \\
\int_{0}^{t} \int_{\Omega}\left(\rho u^{2} u_{x}^{2}+\rho^{\gamma+1} u^{2}\left|u_{x}\right|+p^{2}\left|u_{x}\right|+p u_{x}^{2}\right) d x d s \\
\leq C+C \int_{0}^{t} \max _{x \in \Omega} u^{2}(\cdot, s)\left\|u_{x}(s)\right\|_{\mathcal{L}^{2}}^{2} d s \leq C+C \int_{0}^{t}\left\|u_{x}(s)\right\|_{\mathcal{L}^{2}}^{4} d s, \\
\int_{0}^{t} \int_{\Omega}\left(\left|p u \mathbf{b} \cdot \mathbf{b}_{x}\right|+\left|\mathbf{b} \cdot \mathbf{b}_{t} u_{x}\right|\right) d x d s \leq C \int_{0}^{t} \int_{\Omega}\left(\rho u^{2}+\left|\mathbf{b}_{x}\right|^{2}+\left|\mathbf{b}_{t}\right|^{2}+u_{x}^{2}\right) d x d t \leq C .
\end{gathered}
$$

Therefore, taking $\delta$ appropriately small, we conclude from (2.17)-(2.20) that

$$
\int_{\Omega} u_{x}^{2}(t) d x+\int_{0}^{t} \int_{\Omega} \rho u_{t}^{2} d x d s \leq C+C \int_{0}^{t}\left\|u_{x}(s)\right\|_{\mathcal{L}^{2}}^{4} d s,
$$

where, combined with the fact that $\left\|u_{x}\right\|_{\mathcal{L}^{2}\left(0, T ; \perp^{2}\right)} \leq C$ due to Lemma 2.1, we obtain the first part of Lemma 2.4 by applying Gronwall's lemma and then Sobolev's inequality. Similarly, multiplying (1.4) by $\mathbf{w}_{t}$ and integrating the resulting equation over $\Omega$, we get that

$$
\frac{\mu}{2} \frac{d}{d t} \int_{\Omega}\left|\mathbf{w}_{x}\right|^{2} d x+\frac{1}{2} \int_{\Omega} \rho\left|\mathbf{w}_{t}\right|^{2} d x \leq \int_{\Omega}\left(\frac{1}{2} \rho u^{2}\left|\mathbf{w}_{x}\right|^{2}+\mathbf{b}_{t} \cdot \mathbf{w}_{x}\right) d x-\frac{d}{d t} \int_{\Omega} \mathbf{b} \cdot \mathbf{w}_{x} d x,
$$


Jianwen Zhang

where we have also used Cauchy-Schwarz's inequality. Integration of $(2.22)$ over $(0, t)$ gives

$$
\begin{aligned}
\int_{\Omega}\left|\mathbf{w}_{x}(x, t)\right|^{2} d x & +\int_{0}^{t} \int_{\Omega} \rho\left|\mathbf{w}_{t}\right|^{2} d x d s \leq C+\frac{1}{2} \int_{\Omega}\left|\mathbf{w}_{x}(x, t)\right|^{2} d x \\
& +C \int_{0}^{t} \int_{\Omega}\left(\left|\mathbf{b}_{t}\right|^{2}+\left|\mathbf{w}_{x}\right|^{2}\right) d x d s \leq C+\frac{1}{2} \int_{\Omega}\left|\mathbf{w}_{x}(x, t)\right|^{2} d x,
\end{aligned}
$$

where Lemmas 2.1-2.3 and the first conclusion of this lemma have been used. Therefore, from the above inequality we obtain the second part, and so Lemma 2.4 is proved.

Notice that (1.3), (1.4) can be written as follows:

$$
\rho u_{t}+\rho u u_{x}=G_{x}, \quad \rho \mathbf{w}_{t}+\rho u \mathbf{w}_{x}=\mathbf{K}_{x},
$$

where $G:=\lambda u_{x}-p-|\mathbf{b}|^{2} / 2$ and $\mathbf{K}:=\mu \mathbf{w}_{x}+\mathbf{b}$. Thus, by Lemmas $2.1-2.4$, we see that

$$
\|(G, \mathbf{K})\|_{\mathcal{L}^{\infty}\left(0, T ; \mathcal{L}^{2}\right)}+\left\|\left(G_{x}, \mathbf{K}_{x}\right)\right\|_{\mathcal{L}^{2}\left(0, T ; \mathcal{L}^{2}\right)} \leq C,
$$

which immediately implies

$$
\begin{aligned}
\int_{0}^{T}\left\|u_{x}(t)\right\|_{\mathcal{L}^{\infty}}^{2} d t & \leq C \int_{0}^{T}\left(\|G\|_{\mathcal{L}^{\infty}}^{2}+\|p\|_{\mathcal{L}^{\infty}}^{2}+\|\mathbf{b}\|_{\mathcal{L}^{\infty}}^{4}\right)(t) d t \\
& \leq C \int_{0}^{T}\left(\|G\|_{\mathcal{L}^{2}}^{2}+\left\|G_{x}\right\|_{\mathcal{L}^{2}}^{2}+\|p\|_{\mathcal{L}^{\infty}}^{2}+\|\mathbf{b}\|_{\mathcal{L}^{\infty}}^{4}\right)(t) d t \leq C, \\
\int_{0}^{T}\left\|\mathbf{w}_{x}(t)\right\|_{\mathcal{L}^{\infty}}^{2} d t & \leq C \int_{0}^{T}\left(\|\mathbf{K}\|_{\mathcal{L}^{\infty}}^{2}+\|\mathbf{b}\|_{\mathcal{L}^{\infty}}^{2}\right)(t) d t \\
& \leq C \int_{0}^{T}\left(\|\mathbf{K}\|_{\mathcal{L}^{2}}^{2}+\left\|\mathbf{K}_{x}\right\|_{\mathcal{L}^{2}}^{2}+\|\mathbf{b}\|_{\mathcal{L}^{\infty}}^{2}\right)(t) d t \leq C .
\end{aligned}
$$

Hence, we have the following lemma.

Lemma 2.5. There exists a positive constant $C$, such that

$$
\int_{0}^{T}\left(\left\|u_{x}(t)\right\|_{\mathcal{L}^{\infty}}^{2}+\left\|G_{x}(t)\right\|_{\mathcal{L}^{2}}^{2}\right) d t \leq C, \quad \int_{0}^{T}\left(\left\|\mathbf{w}_{x}(t)\right\|_{\mathcal{L}^{\infty}}^{2}+\left\|\mathbf{K}_{x}(t)\right\|_{\mathcal{L}^{2}}^{2}\right) d t \leq C,
$$

where $G:=\lambda u_{x}-p-|\mathbf{b}|^{2} / 2$ and $\mathbf{K}:=\mu \mathbf{w}_{x}+\mathbf{b}$.

To prove the uniqueness of strong solutions, we still need the following estimates.

Lemma 2.6. The pressure $p(\rho)=R \rho^{r}$ satisfies $\sup _{0 \leq t \leq T}\left\|p_{x}(\cdot, t)\right\|_{\mathfrak{L}^{2}} \leq C$. Furthermore, if the compatibility conditions (1.9), (1.10) hold, then

$$
\sup _{0 \leq t \leq T} \int_{\Omega} \rho\left(u_{t}^{2}+\left|\mathbf{w}_{t}\right|^{2}\right)(x, t) d x+\int_{0}^{T} \int_{\Omega}\left(u_{t x}^{2}+\left|\mathbf{w}_{t x}\right|^{2}\right) d x d t \leq C .
$$


Proof. It follows from the continuity equation (1.2) that $p$ satisfies

$$
p_{t}+p_{x} u+r p u_{x}=0
$$

which, differentiated with respect to $x$, leads to

$$
p_{x t}+p_{x x} u+(\gamma+1) p_{x} u_{x}+\gamma p u_{x x}=0
$$

Multiplying the above equation by $p_{x}$ and integrating over $\Omega$, we deduce that

$$
\begin{aligned}
\frac{d}{d t}\left\|p_{x}(t)\right\|_{\mathcal{L}^{2}}^{2} & \leq C \int_{\Omega}\left(\left|p_{x}\right|^{2}\left|u_{x}\right|+p\left|p_{x}\right|\left|u_{x x}\right|\right)(x, t) d x \\
& \leq C\left(\left\|u_{x}(t)\right\|_{\mathcal{L}^{\infty}}\left\|p_{x}(t)\right\|_{\mathcal{L}^{2}}^{2}+\left\|p_{x}(t)\right\|_{\mathcal{L}^{2}}^{2}+\left\|\mathbf{b}_{x}(t)\right\|_{\mathcal{L}^{2}}^{2}+\left\|G_{x}(t)\right\|_{\mathcal{L}^{2}}^{2}\right),
\end{aligned}
$$

where we have used the inequality

$$
\left\|u_{x x}\right\|_{\mathcal{L}^{2}}^{2} \leq C\left(\left\|G_{x}\right\|_{\mathcal{L}^{2}}^{2}+\left\|p_{x}\right\|_{\mathcal{L}^{2}}^{2}+\left\|\mathbf{b}_{x}\right\|_{\mathcal{L}^{2}}^{2}\right),
$$

which follows from the definition of $G$. Therefore, applying the previous Lemmas 2.1-2.5 and Gronwall's lemma, one has

$$
\sup _{0 \leq t \leq T}\left\|p_{x}(t)\right\|_{\mathcal{L}^{2}} \leq C
$$

which proves the first part of the lemma.

We are now in a position to prove the second part. We first derive the estimate for the longitudinal velocity $u$. To this end, we firstly rewrite (1.3) as

$$
\rho u_{t}+\rho u u_{x}-\lambda u_{x x}+\left(p+\frac{|\mathbf{b}|^{2}}{2}\right)_{x}=0 .
$$

Differentiation of (2.34) with respect to $t$ gives

$$
\rho u_{t t}+\rho u u_{x t}-\lambda u_{x x t}+\left(p+\frac{1}{2}|\mathbf{b}|^{2}\right)_{x t}=-\rho_{t}\left(u_{t}+u u_{x}\right)-\rho u_{t} u_{x}
$$

which, multiplied by $u_{t}$ and integrated by parts over $\Omega$, yields

$$
\frac{1}{2} \frac{d}{d t} \int_{\Omega} \rho u_{t}^{2} d x+\lambda \int_{\Omega} u_{x t}^{2} d x-\int_{\Omega}\left(p+\frac{1}{2}|\mathbf{b}|^{2}\right)_{t} u_{x t} d x=-\int_{\Omega}\left(\rho u\left(u_{t}^{2}+u u_{x} u_{t}\right)_{x}+\rho u_{t}^{2} u_{x}\right) d x
$$

On the other hand, by virtue of (1.2) we have

$$
\begin{aligned}
-\int_{\Omega} p_{t} u_{t x} d x & =\int_{\Omega} p_{x} u u_{t x} d x+\frac{\gamma}{2} \frac{d}{d t} \int_{\Omega} p u_{x}^{2} d x-\frac{\gamma}{2} \int_{\Omega} p_{t} u_{x}^{2} d x \\
& =\frac{\gamma}{2} \frac{d}{d t} \int_{\Omega} p u_{x}^{2} d x+\int_{\Omega} p_{x} u u_{t x} d x+\frac{\gamma}{2} \int_{\Omega}\left(-p u\left(u_{x}^{2}\right)_{x}+(\gamma-1) p u_{x}^{3}\right) d x
\end{aligned}
$$


from which and (2.36) we see that

$$
\begin{aligned}
& \frac{d}{d t} \int_{\Omega}\left(\frac{1}{2} \rho u_{t}^{2}+\frac{\gamma}{2} p u_{x}^{2}\right) d x+\lambda \int_{\Omega} u_{t x}^{2} d x \\
& \quad \leq \int_{\Omega}\left(2 \rho|u|\left|u_{t}\right|\left|u_{t x}\right|+\rho|u|\left|u_{t}\right|\left|u_{x}\right|^{2}+\rho|u|^{2}\left|u_{t}\right|\left|u_{x x}\right|+\rho|u|^{2}\left|u_{x}\right|\left|u_{t x}\right|\right. \\
& \left.\quad \quad+\rho\left|u_{t}\right|^{2}\left|u_{x}\right|+\left|p_{x}\right||u|\left|u_{t x}\right|+\gamma p|u|\left|u_{x}\right|\left|u_{x x}\right|+\frac{\gamma(\gamma-1)}{2} p\left|u_{x}\right|^{3}+|\mathbf{b}|\left|\mathbf{b}_{t}\right|\left|u_{t x}\right|\right) d x \\
& \quad \equiv \sum_{j=1}^{9} I_{j} .
\end{aligned}
$$

Using the previous lemmas and Young's inequality, we can estimate each term on the right-hand side of (2.38) as follows with a small positive constant $\epsilon$ :

$$
\begin{aligned}
& I_{1} \leq 2\|\rho\|_{\mathcal{L}^{\infty}}^{1 / 2}\|u\|_{\mathcal{L}^{\infty}}\left\|\sqrt{\rho} u_{t}\right\|_{\mathcal{L}^{2}}\left\|u_{t x}\right\|_{\mathcal{L}^{2}} \leq \epsilon\left\|u_{t x}\right\|_{\mathcal{L}^{2}}^{2}+C \epsilon^{-1}\left\|\sqrt{\rho} u_{t}\right\|_{\mathcal{L}^{2}}^{2}, \\
& I_{2} \leq\|\rho\|_{\mathcal{L}^{\infty}}^{1 / 2}\|u\|_{\mathcal{L}^{\infty}}\left\|\sqrt{\rho} u_{t}\right\|_{\mathcal{L}^{2}}\left\|u_{x}\right\|_{\mathcal{L}^{2}}\left\|u_{x}\right\|_{\mathcal{L}^{\infty}} \leq C\left\|u_{x}\right\|_{\mathcal{L}^{\infty}}^{2}+C\left\|\sqrt{\rho} u_{t}\right\|_{\mathcal{L}^{2}}^{2}, \\
& I_{3} \leq\|\rho\|_{\mathcal{L}^{\infty}}^{1 / 2}\|u\|_{\mathcal{L}^{\infty}}^{2}\left\|\sqrt{\rho} u_{t}\right\|_{\mathcal{L}^{2}}\left\|u_{x x}\right\|_{\mathcal{L}^{2}} \leq C\left\|u_{x x}\right\|_{\mathcal{L}^{2}}^{2}+C\left\|\sqrt{\rho} u_{t}\right\|_{\mathcal{L}^{2}}^{2}, \\
& I_{4} \leq\|\rho\|_{\mathcal{L}^{\infty}}\|u\|_{\mathcal{L}^{\infty}}^{2}\left\|u_{x}\right\|_{\mathcal{L}^{2}}\left\|u_{t x}\right\|_{\mathcal{L}^{2}} \leq \epsilon\left\|u_{t x}\right\|_{\mathcal{L}^{2}}^{2}+C \epsilon^{-1}, \\
& I_{5} \leq\left\|u_{x}\right\|_{\mathcal{L}^{\infty}}\left\|\sqrt{\rho} u_{t}\right\|_{\mathcal{L}^{2}}^{2} \\
& I_{6} \leq\|u\|_{\mathcal{L}^{\infty}}\left\|p_{x}\right\|_{\mathcal{L}^{2}}\left\|u_{t x}\right\|_{\mathcal{L}^{2}} \leq \epsilon\left\|u_{t x}\right\|_{\mathcal{L}^{2}}^{2}+C \epsilon^{-1}, \\
& I_{7} \leq \gamma\|p\|_{\mathcal{L}^{\infty}}\|u\|_{\mathcal{L}^{\infty}}\left\|u_{x}\right\|_{\mathcal{L}^{2}}\left\|u_{x x}\right\|_{\mathcal{L}^{2}} \leq C+C\left\|u_{x x}\right\|_{\mathcal{L}^{2}}^{2} \\
& I_{8} \leq C\|p\|_{\mathcal{L}^{\infty}}\left\|u_{x}\right\|\left\|_{\mathcal{L}^{\infty}}\right\| u_{x}\left\|_{\mathcal{L}^{2}}^{2} \leq C+C\right\| u_{x} \|_{\mathcal{L}^{\infty}}^{2} \\
& I_{9} \leq\|\mathbf{b}\|_{\mathcal{L}^{\infty}}\left\|\mathbf{b}_{t}\right\|_{\mathcal{L}^{2}}\left\|u_{t x}\right\|_{\mathcal{L}^{2}} \leq \epsilon\left\|u_{t x}\right\|_{\mathcal{L}^{2}}^{2}+C \epsilon^{-1}\left\|\mathbf{b}_{t}\right\|_{\mathcal{L}^{2}}^{2} .
\end{aligned}
$$

Putting the above estimates into (2.38) and taking $\epsilon$ sufficiently small, we arrive at

$$
\begin{aligned}
& \frac{d}{d t} \int_{\Omega}\left(\rho u_{t}^{2}+p u_{x}^{2}\right) d x+\int_{\Omega} u_{t x}^{2} d x \\
& \quad \leq C\left(1+\left\|\sqrt{\rho} u_{t}\right\|_{\mathcal{L}^{2}}^{2}+\left\|u_{x x}\right\|_{\mathcal{L}^{2}}^{2}+\left\|u_{x}\right\|_{\mathcal{L}^{\infty}}^{2}+\left\|\mathbf{b}_{t}\right\|_{\mathcal{L}^{2}}^{2}+\left\|u_{x}\right\|_{\mathcal{L}^{\infty}}\left\|\sqrt{\rho} u_{t}\right\|_{\mathcal{L}^{2}}^{2}\right)
\end{aligned}
$$

so that, using the relation between $G_{x}$ and $u_{x x}$ again, one infers from (2.40) that

$$
\begin{aligned}
& \frac{d}{d t} \int_{\Omega}\left(\rho u_{t}^{2}+p u_{x}^{2}\right) d x+\int_{\Omega} u_{t x}^{2} d x \\
& \quad \leq C\left(1+\left\|\sqrt{\rho} u_{t}\right\|_{\mathcal{L}^{2}}^{2}+\left\|G_{x}\right\|_{\mathcal{L}^{2}}^{2}+\left\|u_{x}\right\|_{\mathcal{L}^{\infty}}^{2}+\left\|\mathbf{b}_{t}\right\|_{\mathcal{L}^{2}}^{2}\right)+C\left\|u_{x}\right\|_{\mathcal{L}^{\infty}}\left\|\sqrt{\rho} u_{t}\right\|_{\mathcal{L}^{2}}^{2}
\end{aligned}
$$


where the first term on the right-hand side of $(2.41)$ is integrable on $(0, T)$ due to the previous lemmas. Thus, integrating (2.41) over $(\tau, t) \subset(0, T)$, we deduce from (1.3) that

$$
\begin{aligned}
\int_{\Omega} \rho u_{t}^{2}(x, t) d x+\int_{\tau}^{t} \int_{\Omega} u_{t x}^{2} d x d s \\
\leq \int_{\Omega} \rho u_{t}^{2}(x, \tau) d x+C\left(1+\int_{0}^{t}\left\|u_{x}(s)\right\|_{\perp^{\infty}}\left\|\sqrt{\rho} u_{t}(s)\right\|_{\mathcal{L}^{2}}^{2} d s\right) \\
=\int_{\Omega} \rho^{-1}\left(\lambda u_{x x}-\left(p+\frac{1}{2}|\mathbf{b}|^{2}\right)_{x}-\rho u u_{x}\right)^{2}(x, \tau) d x \\
\quad+C\left(1+\int_{0}^{t}\left\|u_{x}(s)\right\|_{\mathcal{L}^{\infty}}\left\|\sqrt{\rho} u_{t}(s)\right\|_{\mathcal{L}^{2}}^{2} d s\right) .
\end{aligned}
$$

Letting $\tau \rightarrow 0$ and using the compatibility condition (1.9), we easily obtain from (2.42) that

$$
\int_{\Omega} \rho u_{t}^{2}(x, t) d x+\int_{0}^{t} \int_{\Omega} u_{t x}^{2} d x d s \leq C\left(1+\int_{0}^{t}\left\|u_{x}(s)\right\|_{\mathcal{L}^{\infty}}\left\|\sqrt{\rho} u_{t}(s)\right\|_{\mathcal{L}^{2}}^{2} d s\right),
$$

which, together with $\left\|u_{x}\right\|_{\mathcal{L}^{1}\left(0, T ; \mathcal{L}^{\infty}\right)} \leq C$ and Gronwall's lemma, immediately yields

$$
\sup _{0 \leq t \leq T}\left\|\sqrt{\rho} u_{t}(t)\right\|_{\mathcal{L}^{2}}^{2}+\left\|u_{t x}\right\|_{\mathcal{L}^{2}\left(0, T ; \perp^{2}\right)}^{2} \leq C .
$$

In a same manner as that in the derivation of (2.44), we can show the analogous estimate for the transverse velocity $\mathbf{w}$ by using the previous lemmas, (2.44), and the compatibility condition (1.10) as well. Thus, we complete the proof of Lemma 2.6.

Remark 2.7. From the a priori estimates established above, one sees that the compatibility conditions are used to obtain the second part of Lemma 2.6 only. However, this is crucial in the proof of the uniqueness of strong solutions.

\section{Global existence of strong solutions}

In this section, we prove the global existence of strong solutions to the problem (1.2)-(1.7) by applying the a priori estimates given in the previous section. As usual, we first mollify the initial data to get the existence of smooth approximate solutions. For this purpose, we choose the smooth approximate functions $\rho_{0}^{\epsilon}$ and $\mathbf{b}_{0}^{\epsilon}$ such that

$$
\begin{gathered}
\rho_{0}^{\epsilon} \in C^{2}(\bar{\Omega}), \quad 0<\epsilon \leq \rho_{0}^{\epsilon} \leq\left\|\rho_{0}\right\|_{\rho_{\infty}}+1, \quad \rho_{0}^{\epsilon} \longrightarrow \rho_{0} \quad \text { in } \mathscr{H}^{1}, \\
\mathbf{b}_{0}^{\epsilon} \in C^{2}(\bar{\Omega}), \quad \mathbf{b}_{0}^{\epsilon} \leq\left\|\mathbf{b}_{0}\right\|_{\mathcal{L}^{\infty}}+1, \quad \mathbf{b}_{0}^{\epsilon} \longrightarrow \mathbf{b}_{0} \quad \text { in } \mathscr{L}_{0}^{1} .
\end{gathered}
$$

Let $\left(u_{0}^{\epsilon}, \mathbf{w}_{0}^{\epsilon}\right) \in C_{0}^{1}(\bar{\Omega}) \cap C^{3}(\Omega)$, satisfying $\left(u_{0}^{\epsilon}, \mathbf{w}_{0}^{\epsilon}\right) \rightarrow\left(u_{0}, \mathbf{w}_{0}\right)$ in $\mathscr{\ell}_{0}^{1} \cap \mathscr{\ell}^{2}$, be the unique solution to the boundary value problems

$$
\begin{aligned}
\lambda u_{0 x x}^{\epsilon} & =\left(R\left(\rho_{0}^{\epsilon}\right)^{\gamma}+\frac{1}{2}\left|\mathbf{b}_{0}^{\epsilon}\right|^{2}\right)_{x}+\left(\rho_{0}^{\epsilon}\right)^{1 / 2} f^{\epsilon} \quad \text { in } \Omega,\left.u_{0}^{\epsilon}\right|_{x=0,1}=0, \\
\mu \mathbf{w}_{0 x x}^{\epsilon} & =-\mathbf{b}_{0 x}^{\epsilon}+\left(\rho_{0}^{\epsilon}\right)^{1 / 2} \mathbf{g}^{\epsilon} \quad \text { in } \Omega,\left.\mathbf{w}_{0}^{\epsilon}\right|_{x=0,1}=0,
\end{aligned}
$$


respectively, where

$$
\left(f^{\epsilon}, \mathbf{g}^{\epsilon}\right) \in C_{0}^{2}(\Omega), \quad\left(f^{\epsilon}, \mathbf{g}^{\epsilon}\right) \longrightarrow(f, g) \text { in } \mathcal{L}^{2} .
$$

Thus, with the regularized initial data $\left(\rho_{0}^{e}, u_{0}^{e}, \mathbf{w}_{0}^{\epsilon}, \mathbf{b}_{0}^{\epsilon}\right)$ satisfying the compatibility conditions as above, we can follow the similar arguments as in $[3,9,14]$ (because of $\rho_{0}^{\epsilon} \geq \epsilon$ ) to show that the problems (1.2)-(1.7) admit a global strong solution $\left(\rho^{\epsilon}, u^{\epsilon}, \mathbf{w}^{\epsilon}, \mathbf{b}^{\epsilon}\right)$, which satisfies

$$
0<C(\epsilon) \leq \rho^{\epsilon} \leq C \text { with } C(\epsilon) \text { depending on } \epsilon \text {. }
$$

Applying the a priori estimates obtained in the previous section, we conclude that the approximate solution $\left(\rho^{\epsilon}, \boldsymbol{u}^{\epsilon}, \mathbf{w}^{\epsilon}, \mathbf{b}^{\epsilon}\right)$ satisfies

$$
\begin{aligned}
\sup _{0 \leq t \leq T} & \left(\left\|p^{\epsilon}(t)\right\|_{\mathscr{L}^{1}}+\left\|\left(u_{x}^{\epsilon}, \mathbf{w}_{x}^{\epsilon}, \mathbf{b}_{x}^{\epsilon}\right)(t)\right\|_{\mathcal{L}^{2}}+\left\|\left(\sqrt{\rho^{\epsilon}} u_{t}^{\epsilon}, \sqrt{\rho^{\epsilon}} \mathbf{w}_{t}^{\epsilon}\right)(t)\right\|_{\mathcal{L}^{2}}\right) \\
& +\int_{0}^{T}\left(\left\|\left(u_{x}^{\epsilon}, \mathbf{w}_{x}^{\epsilon}\right)(t)\right\|_{\mathcal{L}^{\infty}}^{2}+\left\|\left(u_{x x}^{\epsilon}, \mathbf{w}_{x x}^{\epsilon}, u_{t x}^{\epsilon}, \mathbf{w}_{t x}^{\epsilon}, \mathbf{b}_{t}, \mathbf{b}_{x x}^{\epsilon}\right)(t)\right\|_{\mathcal{L}^{2}}^{2}\right) d t \leq C,
\end{aligned}
$$

where $C$ depends on the norms of initial data given in Theorem 1.1, but not on $\epsilon$. With the help of (1.2)-(1.5) and (3.5), it is easy to see that

$$
\begin{gathered}
\left\|\rho_{x}^{\epsilon}\right\|_{\mathcal{L}^{\infty}\left(0, T ; \mathcal{L}^{2}\right)} \leq C, \quad\left\|\rho_{t}^{\epsilon}\right\|_{\mathcal{L}^{\infty}\left(0, T ; \mathcal{L}^{2}\right)} \leq C, \\
\left\|u_{x x}^{\epsilon}\right\|_{\mathcal{L}^{\infty}\left(0, T ; \mathcal{L}^{2}\right)} \leq C\left\|\left(\sqrt{\rho^{\epsilon}} u_{t}^{\epsilon}, u_{x}^{\epsilon}, p_{x}^{\epsilon}, \mathbf{b}_{x}^{\epsilon}\right)\right\|_{\mathcal{L}^{\infty}\left(0, T ; \mathcal{L}^{2}\right)} \leq C, \\
\left\|\mathbf{w}_{x x}^{\epsilon}\right\|_{\mathcal{L}^{\infty}\left(0, T ; \mathcal{L}^{2}\right)} \leq C\left\|\left(\sqrt{\rho^{\epsilon}} \mathbf{w}_{t}^{\epsilon}, \mathbf{w}_{x}^{\epsilon}, \mathbf{b}_{x}^{\epsilon}\right)\right\|_{\mathcal{L}^{\infty}\left(0, T ; \mathcal{L}^{2}\right)} \leq C .
\end{gathered}
$$

By the uniform in $\epsilon$ bounds given in (3.5)-(3.8) we conclude that there exists a subsequence of $\left(\rho^{\epsilon}, u^{\epsilon}, \mathbf{w}^{\epsilon}, \mathbf{b}^{\epsilon}\right)$ which converges to a strong solution $(\rho, u, \mathbf{w}, \mathbf{b})$ to the original problem and satisfies (3.5)-(3.8) as well. This completes the proof of Theorem 1.1 except the uniqueness assertion (because of the presence of vacuum), which will be proved in the next section.

\section{Uniqueness and stability of strong solutions}

In this section, we will prove the following stability theorem, which consequently implies the uniqueness of strong solutions. Our proof is inspired by the uniqueness results due to ChoeKim [16, 17] and Desjardins [19] for the isentropic compressible Navier-Stokes equations.

Theorem 4.1. Let $(\rho, u, \mathbf{w}, \mathbf{b})$ and $(\bar{\rho}, \bar{u}, \overline{\mathbf{w}}, \overline{\mathbf{b}})$ be global solutions to problems (1.2)-(1.7) with initial data $\left(\rho_{0}, u_{0}, \mathbf{w}_{0}, \mathbf{b}_{0}\right)$ and $\left(\bar{\rho}_{0}, \bar{u}_{0}, \overline{\mathbf{w}}_{0}, \overline{\mathbf{b}}_{0}\right)$, respectively. If $(\rho, u, \mathbf{w}, \mathbf{b})$ and $(\bar{\rho}, \bar{u}, \overline{\mathbf{w}}, \overline{\mathbf{b}})$ satisfy the regularity given in Theorem 1.1, then for any $t \in(0, T)$,

$$
\begin{aligned}
& \|(\rho-\bar{\rho}, p-\bar{p}, \sqrt{\rho}(u-\bar{u}), \sqrt{\rho}(\mathbf{w}-\overline{\mathbf{w}}), \mathbf{b}-\overline{\mathbf{b}})(t)\|_{\mathcal{L}^{2}}^{2} \\
& \quad+\int_{0}^{t} \exp \left(\int_{S}^{t} F(\tau) d \tau\right)\left\|(u-\bar{u}, \mathbf{w}-\overline{\mathbf{w}}, \mathbf{b}-\overline{\mathbf{b}})_{x}(s)\right\|_{\mathcal{L}^{2}}^{2} d s \\
& \quad \leq \exp \left(\int_{0}^{t} F(s) d s\right)\left\|\left(\rho_{0}-\bar{\rho}_{0}, p_{0}-\bar{p}_{0}, \sqrt{\rho}_{0}\left(u_{0}-\bar{u}_{0}\right), \sqrt{\rho_{0}}\left(\mathbf{w}_{0}-\overline{\mathbf{w}}_{0}\right), \mathbf{b}_{0}-\overline{\mathbf{b}}_{0}\right)\right\|_{\mathcal{L}^{2}}^{2}
\end{aligned}
$$

for some $F(t) \in \mathcal{L}^{1}(0, T)$. Here $p=p(\rho), \bar{p}=p(\bar{\rho})$, and $p_{0}=p\left(\rho_{0}\right), \bar{p}_{0}=p\left(\bar{\rho}_{0}\right)$. 
Proof. From the continuity equation it follows that

$$
(\rho-\bar{\rho})_{t}+(\rho-\bar{\rho})_{x} u+\bar{\rho}_{x}(u-\bar{u})+(\rho-\bar{\rho}) \bar{u}_{x}+\rho(u-\bar{u})_{x}=0 .
$$

Multiplying this by $2(\rho-\bar{\rho})$ and then integrating over $\Omega$, we have

$$
\begin{aligned}
& \frac{d}{d t} \int_{\Omega}|\rho-\bar{\rho}|^{2} d x \\
& \quad \leq \int_{\Omega}|\rho-\bar{\rho}|^{2}\left|u_{x}\right|+2\left|\bar{\rho}_{x}\right|\left|\rho-\bar{\rho}\left\|u-\bar{u}|+2| \rho-\left.\bar{\rho}\right|^{2}\left|\bar{u}_{x}\right|+2 \rho\left|\rho-\bar{\rho} \|(u-\bar{u})_{x}\right| d x\right.\right. \\
& \quad \leq\left(\left\|u_{x}\right\|_{\mathcal{L}^{\infty}}+2\left\|\bar{u}_{x}\right\|_{\mathcal{L}^{\infty}}\right)\|\rho-\bar{\rho}\|_{\mathcal{L}^{2}}^{2}+2\left(\left\|\bar{\rho}_{x}\right\|_{\mathcal{L}^{2}}+\|\rho\|_{\mathcal{L}^{\infty}}\right)\|\rho-\bar{\rho}\|_{\mathcal{L}^{2}}\left\|(u-\bar{u})_{x}\right\|_{\mathcal{L}^{2},}
\end{aligned}
$$

where we have used the inequality $\|(u-\bar{u})\|_{\mathcal{L}_{\infty}} \leq\left\|(u-\bar{u})_{x}\right\|_{\mathcal{L}^{2}}$. Thus, by Cauchy-Schwarz's inequality, one has

$$
\frac{d}{d t}\|\rho-\bar{\rho}\|_{\mathcal{L}^{2}}^{2} \leq \epsilon\left\|(u-\bar{u})_{x}\right\|_{\mathcal{L}^{2}}^{2}+C \epsilon^{-1} A(t)\|\rho-\bar{\rho}\|_{\mathcal{L}^{2}}^{2}
$$

where $A(t):=\left(\left\|u_{x}\right\|_{\mathcal{L}^{\infty}}+\left\|\bar{u}_{x}\right\|_{\mathcal{L}_{\infty}}+\left\|\bar{\rho}_{x}\right\|_{\mathcal{L}^{2}}^{2}+\|\rho\|_{\mathcal{L}^{\infty}}^{2}\right) \in \mathcal{L}^{1}(0, T)$.

By virtue of the equations satisfied by $u$ and $\bar{u}$, we easily deduce that

$$
\begin{aligned}
& \rho(u-\bar{u})_{t}+\rho u(u-\bar{u})_{x}-\lambda(u-\bar{u})_{x x} \\
& \quad=-(\rho-\bar{\rho})\left(\bar{u}_{t}+\overline{u u}_{x}\right)-\rho(u-\bar{u}) \bar{u}_{x}-(p-\bar{p})_{x}-\frac{1}{2}\left(|\mathbf{b}|^{2}-|\overline{\mathbf{b}}|^{2}\right)_{x}
\end{aligned}
$$

which, multiplied by $(u-\bar{u})$ and integrated by parts over $\Omega$, gives

$$
\begin{aligned}
& \frac{1}{2} \frac{d}{d t} \int_{\Omega} \rho|u-\bar{u}|^{2} d x+\lambda \int_{\Omega}\left|(u-\bar{u})_{x}\right|^{2} d x \\
& \leq\left(\left\|\bar{u}_{t}\right\|_{\mathcal{L}^{2}}+\|\bar{u}\|_{\mathcal{L}^{\infty}}\left\|\bar{u}_{x}\right\|_{\mathcal{L}^{2}}\right)\|\rho-\bar{\rho}\|_{\mathcal{L}^{2}}\|u-\bar{u}\|_{\mathcal{L}^{\infty}}+\|\sqrt{\rho}(u-\bar{u})\|_{\mathcal{L}^{2}}^{2}\left\|\bar{u}_{x}\right\|_{\mathcal{L}^{\infty}} \\
& \quad+\|p-\bar{p}\|_{\mathcal{L}^{2}}\left\|(u-\bar{u})_{x}\right\|_{\mathcal{L}^{2}}+\frac{1}{2}\left(\|\mathbf{b}\|_{\mathcal{L}^{\infty}}+\|\overline{\mathbf{b}}\|_{\mathcal{L}^{\infty}}\right)\|\mathbf{b}-\overline{\mathbf{b}}\|_{\mathcal{L}^{2}}\left\|(u-\bar{u})_{x}\right\|_{\mathcal{L}^{2}},
\end{aligned}
$$

so that we have by using Sobolev's inequality and Cauchy-Schwarz's inequality that

$$
\begin{aligned}
& \frac{d}{d t}\|\sqrt{\rho}(u-\bar{u})\|_{\mathcal{L}^{2}}^{2}+\left\|(u-\bar{u})_{x}\right\|_{\mathcal{L}^{2}}^{2} \\
& \quad \leq B(t)\left(\|\rho-\bar{\rho}\|_{\mathcal{L}^{2}}^{2}+\|\sqrt{\rho}(u-\bar{u})\|_{\mathcal{L}^{2}}^{2}+\|p-\bar{p}\|_{\mathcal{L}^{2}}^{2}+\|\mathbf{b}-\overline{\mathbf{b}}\|_{\mathcal{L}^{2}}^{2}\right),
\end{aligned}
$$

where $B(t):=C\left(\left\|\bar{u}_{t}\right\|_{\mathcal{L}^{2}}^{2}+\|\bar{u}\|_{\mathcal{L}^{\infty}}^{2}\left\|\bar{u}_{x}\right\|_{\mathcal{L}^{2}}^{2}+\left\|\bar{u}_{x}\right\|_{\mathcal{L}^{\infty}}+\|\mathbf{b}\|_{\mathcal{L}^{\infty}}^{2}+\|\overline{\mathbf{b}}\|_{\mathcal{L}^{\infty}}^{2}\right) \in \mathcal{L}^{1}(0, T)$.

Proceeding the similar argument as that in the derivation of (4.7), we also have

$$
\begin{aligned}
\frac{d}{d t} \| & \sqrt{\rho}(\mathbf{w}-\overline{\mathbf{w}})\left\|_{\mathcal{L}^{2}}^{2}+\right\|(\mathbf{w}-\overline{\mathbf{w}})_{x} \|_{\mathcal{L}^{2}}^{2} \\
& \leq C(t)\left(\|\rho-\bar{\rho}\|_{\mathcal{L}^{2}}^{2}+\|\sqrt{\rho}(u-\bar{u})\|_{\mathcal{L}^{2}}^{2}+\|\sqrt{\rho}(\mathbf{w}-\overline{\mathbf{w}})\|_{\mathcal{L}^{2}}^{2}+\|\mathbf{b}-\overline{\mathbf{b}}\|_{\mathcal{L}^{2}}^{2}\right),
\end{aligned}
$$


where $C(t):=C\left(\left\|\overline{\mathbf{w}}_{t}\right\|_{\mathcal{L}^{2}}^{2}+\|\bar{u}\|_{\mathcal{L}^{\infty}}^{2}\left\|\overline{\mathbf{w}}_{x}\right\|_{\mathcal{L}^{2}}^{2}+\left\|\overline{\mathbf{w}}_{x}\right\|_{\mathcal{L}^{\infty}}\right) \in \mathcal{L}^{1}(0, T)$.

Furthermore, it follows from the equations for the magnetic fields $\mathbf{b}$ and $\overline{\mathbf{b}}$ that

$$
(\mathbf{b}-\overline{\mathbf{b}})_{t}+u(\mathbf{b}-\overline{\mathbf{b}})_{x}+(u-\bar{u}) \overline{\mathbf{b}}_{x}+(u-\bar{u})_{x} \mathbf{b}+\bar{u}_{x}(\mathbf{b}-\overline{\mathbf{b}})-(\mathbf{w}-\overline{\mathbf{w}})_{x}=v(\mathbf{b}-\overline{\mathbf{b}})_{x x}
$$

which, multiplied by $2(\mathbf{b}-\overline{\mathbf{b}})$ and integrated by parts over $\Omega$, gives

$$
\begin{aligned}
& \frac{d}{d t}\|\mathbf{b}-\overline{\mathbf{b}}\|_{\mathcal{L}^{2}}^{2}+2 v\left\|(\mathbf{b}-\overline{\mathbf{b}})_{x}\right\|_{\mathcal{L}^{2}}^{2} \\
& \leq\left(\left\|u_{x}\right\|_{\mathcal{L}^{\infty}}+2\left\|\bar{u}_{x}\right\|_{\mathcal{L}^{\infty}}\right)\|\mathbf{b}-\overline{\mathbf{b}}\|_{\mathcal{L}^{2}}^{2}+2\left\|\overline{\mathbf{b}}_{x}\right\|_{\mathcal{L}^{2}}\|\mathbf{b}-\overline{\mathbf{b}}\|_{\mathcal{L}^{2}}\|u-\bar{u}\|_{\mathcal{L}^{\infty}} \\
& \quad+2\|\mathbf{b}\|_{\mathcal{L}^{\infty}}\|\mathbf{b}-\overline{\mathbf{b}}\|_{\mathcal{L}^{2}}\left\|(u-\bar{u})_{x}\right\|_{\mathcal{L}^{2}}+2\|\mathbf{b}-\overline{\mathbf{b}}\|_{\mathcal{L}^{2}}\left\|(\mathbf{w}-\overline{\mathbf{w}})_{x}\right\|_{\mathcal{L}^{2}} \\
& \leq \epsilon\left(\left\|(u-\bar{u})_{x}\right\|_{\mathcal{L}^{2}}^{2}+\left\|(\mathbf{w}-\overline{\mathbf{w}})_{x}\right\|_{\mathcal{L}^{2}}^{2}\right)+C \epsilon^{-1} D(t)\|\mathbf{b}-\overline{\mathbf{b}}\|_{\mathcal{L}^{2}}^{2}
\end{aligned}
$$

where $D(t):=\left(1+\left\|u_{x}\right\|_{\mathcal{L}^{\infty}}+\left\|\bar{u}_{x}\right\|_{\mathcal{L}^{\infty}}+\left\|\overline{\mathbf{b}}_{x}\right\|_{\mathcal{L}^{2}}^{2}+\|\mathbf{b}\|_{\mathcal{L}^{\infty}}^{2}\right) \in \mathcal{L}^{1}(0, T)$.

Finally, from the continuity equations for $\rho$ and $\bar{\rho}$, it is easy to see that

$$
(p-\bar{p})_{t}+(p-\bar{p})_{x} u+\bar{p}_{x}(u-\bar{u})+\gamma(p-\bar{p}) \bar{u}_{x}+\gamma p(u-\bar{u})_{x}=0,
$$

and hence we obtain in a manner similar to the derivation of (4.4) that

$$
\begin{aligned}
\frac{d}{d t}\|p-\bar{p}\|_{\mathcal{L}^{2}}^{2} \leq & C\left(\left\|u_{x}\right\|_{\mathcal{L}^{\infty}}+\left\|\bar{u}_{x}\right\|_{\mathcal{L}^{\infty}}\right)\|p-\bar{p}\|_{\mathcal{L}^{2}}^{2} \\
& +C\left\|\bar{p}_{x}\right\|_{\mathcal{L}^{2}}\|p-\bar{p}\|_{\mathcal{L}^{2}}\|u-\bar{u}\|_{\mathcal{L}^{\infty}}+C\|p\|_{\mathcal{L}^{\infty}}\|p-\bar{p}\|_{\mathcal{L}^{2}}\left\|(u-\bar{u})_{x}\right\|_{\mathcal{L}^{2}} \\
\leq & \epsilon\left\|(u-\bar{u})_{x}\right\|_{\mathcal{L}^{2}}^{2}+C \epsilon^{-1} E(t)\|p-\bar{p}\|_{\mathcal{L}^{2},}^{2}
\end{aligned}
$$

where $E(t):=\left(\left\|u_{x}\right\|_{\mathcal{L}^{\infty}}+\left\|\bar{u}_{x}\right\|_{\mathcal{L}^{\infty}}+\left\|\bar{p}_{x}\right\|_{\mathcal{L}^{2}}^{2}+\|p\|_{\mathcal{L}^{\infty}}^{2}\right) \in \mathcal{L}^{1}(0, T)$.

Summing up (4.4)-(4.12) and choosing $\epsilon$ appropriately small, we obtain Theorem 4.1 with $F(t)=C(A(t)+B(t)+C(t)+D(t)+E(t)) \in \mathcal{L}^{1}(0, T)$ by applying Gronwall's lemma.

\section{Acknowledgment}

This work is partly supported by NSFC (Grant no. 10501037 and no. 10601008).

\section{References}

[1] J. A. Bittencourt, Fundamentals of Plasma Physics, Spinger, New York, NY, USA, 3rd edition, 2004.

[2] H. Cabannes, Theoretical Magnetofluiddynamics, Academic Press, New York, NY, USA, 1970.

[3] G.-Q. Chen and D. Wang, "Global solutions of nonlinear magnetohydrodynamics with large initial data," Journal of Differential Equations, vol. 182, no. 2, pp. 344-376, 2002.

[4] A. F. Kulikovskiy and G. A. Lyubimov, Magnetohydrodynamics, Addison-Wasley, Reading, Mass, USA, 1965.

[5] L. D. Landau, E. M. Lifshitz, and L. P. Pitaevskii, Electrodynamics of Continuous Media, ButterworthHeinemann, Oxford, UK, 2nd edition, 1999.

[6] R. Balescu, Transport Processes in Plasmas I: Classical Transport Theory, North-Holland, New York, NY, USA, 1988. 
[7] E. Becker, Gasdynamik, vol. 6 of Leitfäden der Angewandten Mathematik und Mechanik, B. G. Teubner Verlagsgesellschaft, Stuttgart, Germany, 1966.

[8] M. Brio and C. C. Wu, "An upwind differencing scheme for the equations of ideal magnetohydrodynamics," Journal of Computational Physics, vol. 75, no. 2, pp. 400-422, 1988.

[9] G.-Q. Chen and D. Wang, "Existence and continuous dependence of large solutions for the magnetohydrodynamic equations," Zeitschrift für Angewandte Mathematik und Physik, vol. 54, no. 4, pp. 608-632, 2003.

[10] J. Fan, S. Jiang, and G. Nakamura, "Vanishing shear viscosity limit in the magnetohydrodynamic equations," Communications in Mathematical Physics, vol. 270, no. 3, pp. 691-708, 2007.

[11] S. Kawashima and M. Okada, "Smooth global solutions for the one-dimensional equations in magnetohydrodynamics," Proceedings of the Japan Academy. Series A, vol. 58, no. 9, pp. 384-387, 1982.

[12] A. V. Kazhikhov and Sh. Smagulov, "Well-posedness and approximation methods for a model of magnetogasdynamics," Izvestiya Akademii Nauk Kazakhskoŭ SSR. Seriya Fiziko-Matematicheskaya, no. 5, pp. 17-19, 1986.

[13] S. S. Smagulov, A. A. Durmagambetov, and D. A. Iskenderova, "The Cauchy problems for equations of magnetogasdynamics," Differential Equations, vol. 29, no. 2, pp. 278-288, 1993.

[14] D. Wang, "Large solutions to the initial-boundary value problem for planar magnetohydrodynamics," SIAM Journal on Applied Mathematics, vol. 63, no. 4, pp. 1424-1441, 2003.

[15] V. V. Shelukhin, "A shear flow problem for the compressible Navier-Stokes equations," International Journal of Non-Linear Mechanics, vol. 33, no. 2, pp. 247-257, 1998.

[16] H. J. Choe and H. Kim, "Strong solutions of the Navier-Stokes equations for isentropic compressible fluids," Journal of Differential Equations, vol. 190, no. 2, pp. 504-523, 2003.

[17] H. J. Choe and H. Kim, "Global existence of the radially symmetric solutions of the Navier-Stokes equations for the isentropic compressible fluids," Mathematical Methods in the Applied Sciences, vol. 28, no. 1, pp. 1-28, 2005.

[18] T. Luo, Z. Xin, and T. Yang, "Interface behavior of compressible Navier-Stokes equations with vacuum," SIAM Journal on Mathematical Analysis, vol. 31, no. 6, pp. 1175-1191, 2000.

[19] B. Desjardins, "Regularity of weak solutions of the compressible isentropic Navier-Stokes equations," Communications in Partial Differential Equations, vol. 22, no. 5-6, pp. 977-1008, 1997. 